\title{
Existence, uniqueness and asymptotic behavior of solutions for a nonclassical diffusion equation with delay
}

\author{
T. Caraballo and A. M. Márquez-Durán \\ Communicated by Yuncheng You, received March 21, 2013.
}

\begin{abstract}
A nonclassical nonautonomous diffusion equation with delay is analyzed. First, we prove the existence and uniqueness of solutions by using the Galerkin approximations and the energy method. Next, we prove the existence and eventual uniqueness of stationary solutions, as well as their exponential stability. We emphasize that the assumptions imposed on the delay term include, in particular, the case of measurable variable delays.
\end{abstract}

\section{Contents}

1. Introduction 267

2. Preliminaries 268

3. Existence and uniqueness of solution 269

4. Stationary solutions and their stability 276

$\begin{array}{ll}\text { References } & 280\end{array}$

\section{Introduction}

In this paper we consider the following nonautonomous nonclassical diffusion problem:

$$
\left\{\begin{array}{l}
\frac{\partial u}{\partial t}-\Delta\left(\frac{\partial u}{\partial t}\right)-\Delta u=g\left(t, u_{t}\right) \text { in }(\tau,+\infty) \times \Omega, \\
u=0 \text { on }(\tau,+\infty) \times \Gamma \\
u(t, x)=\phi(t-\tau, x), t \in[\tau-h, \tau], x \in \Omega
\end{array}\right.
$$

1991 Mathematics Subject Classification. 35.

Key words and phrases. Diffusion equation with delay, existence and uniqueness.

Partly supported by FEDER and Ministerio de Economía y Competitividad (Spain) under project MTM2011-22411, and Junta de Andalucía project FQM314. 
where $\Omega$ is an open bounded set of $\mathbb{R}^{n}, \tau \in \mathbb{R}$ is the initial time, $g$ is an external force depending on $t$ and $u_{t}$, where for each $t \geq \tau$, we denote by $u_{t}$ the function defined on $[-h, 0]$ by the relation $u_{t}(s)=u(t+s), s \in[-h, 0]$, with $h>0$ a fixed time, and $\phi$ is a given function defined on $[-h, 0] \times \Omega$.

This type of nonclassical parabolic equations are often used to model physical phenomena, such as non-Newtonian flows, soil mechanics, heat conduction, etc (see, e.g., $[1,2,6,7,8,10,11])$. In this paper we are interested in the case in which some kind of delay is taken into account in the forcing term. To be more precise, we will be interested in the case in which a bounded general delay is considered in the equation. This is an important variant of the nondelay case because there are many situations in which the evolution of the model is determined not only by the present state of the system but for its past history.

In Section 2 we will establish the problem and the necessary preliminaries to handle our problem. The existence and uniqueness of solution of our problem is proved in Section 3 by using the Galerkin approximations and the energy equality. Finally, in Section 4 we prove the existence of stationary solutions of our problem, and we also analyze the asymptotic behaviour of such stationary solutions, by establishing some sufficient conditions ensuring their exponential stability.

\section{Preliminaries}

We consider the following usual spaces $H=L^{2}(\Omega)$ with inner product $(\cdot, \cdot)$ and associate norm $|\cdot|$, and $V=H_{0}^{1}(\Omega)$ with scalar product $((\cdot, \cdot))=\left(A^{1 / 2} u, A^{1 / 2} v\right)$, for $u, v \in V$, and associate norm $\|\cdot\|$, where $A u=-\Delta u$ for any $u \in D(A)$ with $D(A)=\{u \in V: A u \in H\}=H_{0}^{1}(\Omega) \cap H^{2}(\Omega)$.

We denote $C_{H}=C([-h, 0] ; H)$ with norm $|\phi|_{C_{H}}=\sup _{s \in[-h, 0]}|\phi(s)|, C_{V}=$ $C([-h, 0] ; V)$, with norm $|\phi|_{C_{V}}=\sup _{s \in[-h, 0]}\|\phi(s)\|$, and $\mathbb{R}_{+}=(0,+\infty)$.

For the delay term, we assume that $g:(\tau,+\infty) \times C_{H} \rightarrow H$ and satisfies:

g1) for any $\xi \in C_{H}$, the mapping $\mathbb{R}_{+} \ni t \mapsto g(t, \xi) \in H$ is measurable,

g2) there exists a nondecreasing function $L_{g}:(\tau,+\infty) \rightarrow(\tau,+\infty)$, such that for all $R>0$ if $|\xi|_{C_{H}},|\eta|_{C_{H}} \leq R$, then

$$
|g(t, \xi)-g(t, \eta)| \leq L_{g}(R)|\xi-\eta|_{C_{H}},
$$

for all $t \in(\tau,+\infty)$, and

g3) there exist a constant $C_{g}>0$ and a nonnegative function $f \in L^{1}(\tau, T)$, for all $T>\tau$, such that for any $\xi \in C_{H}$,

$$
|g(t, \xi)|^{2} \leq C_{g}|\xi|_{C_{H}}^{2}+f(t), \quad \text { for all } t \in(\tau,+\infty) .
$$

Finally, we suppose that $\phi \in C_{H}$.

REMARK 2.1. Consider a globally Lipschitz function $G: H \rightarrow H$, with Lipschitz constant $L_{G}>0$, and a measurable function $\rho: \mathbb{R} \rightarrow[0, h]$.

Then, it is not difficult to check that the operator $g: \mathbb{R} \times C_{H} \rightarrow H$, defined by

$$
\mathbb{R} \times C_{H} \ni(t, \xi) \mapsto g(t, \xi):=G(\xi(-\rho(t)))
$$

satisfies assumptions g1)-g3).

Observe that the only assumption imposed on $\rho$ is measurability, in contrast with the condition $\rho \in C^{1}$, with derivative $\rho^{\prime}(t) \leq \rho_{*}<1$ appearing in many papers 
published on delay differential equations (see e.g. [4] for a nonclassical diffusion model with delays).

The example of delay term described above can be generalized in several senses. The most straightforward generalization is to take into account more than one delay term in the problem, and to allow $G$ to depend on time. Namely, consider $m$ measurable functions $\rho_{i}: \mathbb{R} \rightarrow[0, h]$ for $i=1, \ldots, m$, a measurable mapping $G: \mathbb{R}_{+} \times H^{m} \rightarrow H$ such that $G(t, \cdot)$ is locally Lipschitz and sublinear in $H^{m}$ uniformly with respect to time. Then, consider $g: \mathbb{R} \times C_{H} \rightarrow H$ given by $g(t, \xi):=G\left(t, \xi\left(-\rho_{1}(t)\right), \ldots, \xi\left(-\rho_{m}(t)\right)\right)$. This operator $g$ also satisfies conditions g1) - g3).

Another example of operator satisfying assumptions (g1)-(g3) is given below.

We consider $g: \mathbb{R} \times C_{H} \rightarrow H$ defined as follows:

$$
g(t, \xi):=\int_{-h}^{0} G(t, s, \xi(s)) d s \quad \forall t \in \mathbb{R}, \forall \xi \in C_{H},
$$

where the function $G: \mathbb{R} \times(-h, 0) \times \mathbb{R} \rightarrow \mathbb{R}$ satisfies the following assumptions:

(a) $G(t, s, 0)=0$ for all $(t, s) \in \mathbb{R} \times(-h, 0)$.

(b) There exists a function $\kappa:(-h, 0) \rightarrow(0,+\infty)$ which belongs to $L^{2}(-h, 0)$ and such that

$\|G(t, s, u)-G(t, s, v)\|_{\mathbb{R}} \leq \kappa(s)\|u-v\|_{\mathbb{R}}, \quad \forall u, v \in \mathbb{R},(t, s) \in \mathbb{R} \times(-h, 0)$,

Namely, the operator $g$ defines an element of $H$ in the following way:

$$
g(t, \xi)(x)=\int_{-\infty}^{0} G(t, s, \xi(s)(x)) d s, \quad \forall x \in \Omega .
$$

One can check now that $g$ satisfies assumption (g3), and using (a) above, we obtain that it is well defined as a map with values in $H$.

\section{Existence and uniqueness of solution}

In this section we establish existence and uniqueness of solution for (1). But, before studying (1), we consider the autonomous equation $u+A u=g$. From the Lax-Milgram lemma, we know that for each $g \in V^{\prime}$ there exists a unique $u_{g} \in V$ such that

$$
u_{g}+A u_{g}=g .
$$

The mapping $C: u \in V \rightarrow u+A u \in V^{\prime}$ is linear and bijective, with $C^{-1} g=u_{g}$. From (2), one has $\left|u_{g}\right|^{2}+\left\|u_{g}\right\|^{2} \leq\|g\|_{*}\left\|u_{g}\right\|$, and in particular, $\left\|u_{g}\right\| \leq\|g\|_{*}$, i.e.,

$$
\left\|C^{-1} g\right\| \leq\|g\|_{*}, \quad \forall g \in V^{\prime} .
$$

Observe that, by the definition of $D(A)$, we also have that $C^{-1}(H)=D(A)$, and reasoning as for the obtention of (3), we deduce that

$$
\left|A u_{g}\right|=\left|g-u_{g}\right| \leq 2|g|, \quad \forall g \in H .
$$

Let us first define the concept of solution that we will work with.

Definition 3.1. A weak solution of (1) is a function $u \in C([-h, T] ; H) \cap$ $L^{2}(\tau, T ; V)$ for all $T>\tau$, with $u(t)=\phi(t-\tau)$ for all $t \in[\tau-h, \tau]$ and such that for all $w \in V$,

$$
\frac{d}{d t}(u(t), w)+\left(\left(\frac{d}{d t} u(t), w\right)\right)+((u(t), w))=\left(g\left(t, u_{t}\right), w\right), \quad \text { a.e. in }(\tau,+\infty),
$$


or, equivalently,

$$
\frac{d}{d t}(u(t)+A u(t))+A u(t)=g\left(t, u_{t}\right), \quad \text { in } \mathcal{D}^{\prime}\left(\tau,+\infty ; V^{\prime}\right) .
$$

REMARK 3.2. If $u \in C([-h, T] ; H) \cap L^{2}(\tau, T ; V)$ for all $T>\tau$ and satisfies (5), then the function $v$ defined by

$$
v(t)=u(t)+A u(t), \quad t>\tau,
$$

belongs to $L^{2}\left(\tau, T ; V^{\prime}\right)$ for all $T>\tau$, and, by $\left.g 3\right), v^{\prime} \in L^{1}\left(\tau, T ; V^{\prime}\right)$ for all $T>\tau$. Consequently, $v \in C\left([\tau,+\infty) ; V^{\prime}\right)$, and therefore, by (3), $u \in C([\tau,+\infty) ; V)$. Moreover, again by $g 3)$ and (5), $v^{\prime} \in L^{2}\left(\tau, T ; V^{\prime}\right)$ for all $T>\tau$, and therefore, as $u^{\prime}=C^{-1} v^{\prime}$, we deduce that $u^{\prime} \in L^{2}(\tau, T ; V)$ for all $T>\tau$. From these considerations, it is clear that $u$ is a weak solution to (1) if and only if $u \in C([\tau,+\infty) ; V), u^{\prime} \in$ $L^{2}(\tau, T ; V)$ for all $T>\tau$, and

$$
u(t)+A u(t)+\int_{\tau}^{t} A u(s) d s=u(\tau)+A u(\tau)+\int_{\tau}^{t} g\left(s, u_{s}\right) d s\left(\text { equality in } V^{\prime}\right),
$$

for all $t \geq \tau$.

REMARK 3.3. If $u$ is a weak solution of (1), then $u$ satisfies the following energy equality:

$$
\begin{aligned}
& |u(t)|^{2}+\|u(t)\|^{2}+2 \int_{s}^{t}\|u(r)\|^{2} d r \\
& \quad=|u(s)|^{2}+\|u(s)\|^{2}+2 \int_{s}^{t}\left(g\left(r, u_{r}\right), u(r)\right) d r \quad \forall s, t \in[0, \infty) .
\end{aligned}
$$

Our main result in this section is the following.

Theorem 3.4. Assume that $g$ satisfies assumptions g1)-g3), and $\phi \in C_{H}$ with $\phi(0) \in V$. Then, there exists a unique weak solution $u=u(\cdot ; \tau, \phi)$ of (1), which satisfies in addition that

$$
u \in C([\tau, T] ; V)
$$

for all $T>\tau$. And, if $\phi \in C_{V}$, then $u \in C([\tau-h, T] ; V)$.

Proof. For simplicity, we will argue in the case $\tau=0$. The general case is similar. We split the proof of existence into two steps.

Step 1: A Galerkin scheme. First a priori estimates. Let us consider $\left\{v_{j}\right\} \subset V$, the orthonormal basis of $H$ of all the eigenfunctions of the operator $A$. Denote $V_{m}=\operatorname{span}\left[v_{1}, \ldots, v_{m}\right]$ and consider the projector $P_{m} u=\sum_{j=1}^{m}\left(u, v_{j}\right) v_{j}$.

Define also

$$
u^{m}(t)=\sum_{j=1}^{m} \alpha_{m, j}(t) v_{j},
$$

where the upper script $m$ will be used instead of $(m)$ since no confusion is possible with powers of $u$, and where the coefficients $\alpha_{m, j}$ are required to satisfy the following system of ordinary differential equations:

$$
\begin{aligned}
\frac{d}{d t}\left(u^{m}(t), v_{j}\right) & +\frac{d}{d t}\left(\left(u^{m}(t), v_{j}\right)\right)+\left(\left(u^{m}(t), v_{j}\right)\right) \\
= & \left(g\left(t, u_{t}^{m}\right), v_{j}\right), \quad \text { a.e. } t>0, \quad 1 \leq j \leq m,
\end{aligned}
$$


and the initial condition $u^{m}(s)=P_{m} \phi(s)$ for $s \in[-h, 0]$.

In principle, the above system of ordinary functional differential equations (8) possesses a unique local solution defined in $\left[0, t_{m}\right)$, with $0<t_{m} \leq \infty$ (see [3]).

Let us prove that the solutions do exist for all time $t \in[0,+\infty)$.

Let us fix $0<T<t_{m}$. Multiplying (8) by $\alpha_{m_{j}}(t)$ and summing in $j$, we obtain for a.e. $t \in(0, T)$,

$$
\begin{aligned}
\frac{1}{2} \frac{d}{d t}\left|u^{m}(t)\right|^{2}+\frac{1}{2} \frac{d}{d t}\left\|u^{m}(t)\right\|^{2}+\left\|u^{m}(t)\right\|^{2} & =\left(g\left(t, u_{t}^{m}\right), u^{m}(t)\right) \\
& \leq\left|g\left(t, u_{t}^{m}\right)\right|\left|u^{m}(t)\right|,
\end{aligned}
$$

and therefore, using Young's inequality, and taking into account g3), we deduce that

$$
\frac{d}{d t}\left(\left|u^{m}(t)\right|^{2}+\left\|u^{m}(t)\right\|^{2}\right) \leq\left(2 \lambda_{1}\right)^{-1}\left[C_{g}\left|u_{t}^{m}\right|_{C_{H}}^{2}+f(t)\right] \quad \text { a.e. } t \in(0, T) .
$$

Hence, integrating between 0 and $t$, and taking into account that $\phi(0) \in V$, we have

$$
\left|u^{m}(t)\right|^{2}+\left\|u^{m}(t)\right\|^{2} \leq|\phi(0)|^{2}+\|\phi(0)\|^{2}+\left(2 \lambda_{1}\right)^{-1} \int_{0}^{t}\left(C_{g}\left|u_{s}^{m}\right|_{C_{H}}^{2}+f(s)\right) d s,
$$

for all $t \in[0, T]$.

On the one hand, if $t \leq h$,

$$
\begin{aligned}
\left|u_{t}^{m}\right|_{C_{H}}^{2} \leq \max \left\{\sup _{\theta} \mid \phi(-h,-t]\right. & |\phi(\theta+t)|^{2}, \sup _{\theta \in[-t, 0]}\left[|\phi(0)|^{2}\right. \\
& \left.\left.+\|\phi(0)\|^{2}+\frac{1}{2 \lambda_{1}} \int_{0}^{t+\theta}\left(C_{g}\left|u_{s}^{m}\right|_{C_{H}}^{2}+f(s)\right) d s\right]\right\} .
\end{aligned}
$$

But,

$$
\begin{aligned}
\sup _{\theta \in[-h,-t]}|\phi(\theta+t)| & \leq \sup _{\theta \in[-h, 0]}|\phi(\theta)| \\
& =|\phi|_{C_{H}},
\end{aligned}
$$

and

$$
\sup _{\theta \in[-t, 0]} \int_{0}^{t+\theta}\left(C_{g}\left|u_{s}^{m}\right|_{C_{H}}^{2}+f(s)\right) d s \leq \int_{0}^{t}\left(C_{g}\left|u_{s}^{m}\right|_{C_{H}}^{2}+f(s)\right) d s .
$$

Therefore, from these inequalities we can deduce, if $t \leq h$,

$$
\left|u_{t}^{m}\right|_{C_{H}}^{2} \leq|\phi|_{C_{H}}^{2}+\|\phi(0)\|^{2}+\frac{1}{2 \lambda_{1}} \int_{0}^{t}\left(C_{g}\left|u_{s}^{m}\right|_{C_{H}}^{2}+f(s)\right) d s
$$

If $t>h$,

$$
\left|u_{t}^{m}\right|_{C_{H}}^{2} \leq \sup _{\theta \in[-h, 0]}\left[|\phi(0)|^{2}+\|\phi(0)\|^{2}+\frac{1}{2 \lambda_{1}} \int_{0}^{t+\theta}\left(C_{g}\left|u_{s}^{m}\right|_{C_{H}}^{2}+f(s)\right) d s\right] .
$$

Therefore, we can conclude that

$\left|u_{t}^{m}\right|_{C_{H}}^{2} \leq|\phi|_{C_{H}}^{2}+\|\phi(0)\|^{2}+\left(2 \lambda_{1}\right)^{-1} \int_{0}^{t}\left(C_{g}\left|u_{s}^{m}\right|_{C_{H}}^{2}+f(s)\right) d s, \quad$ for all $t \in[0, T]$, 
and, by the Gronwall lemma we have

$$
\left|u_{t}^{m}\right|_{C_{H}}^{2} \leq e^{C_{g}\left(2 \lambda_{1}\right)^{-1} t}\left[|\phi|_{C_{H}}^{2}+\|\phi(0)\|^{2}+\left(2 \lambda_{1}\right)^{-1} \int_{0}^{t} f(s) d s\right],
$$

for all $t \in[0, T]$.

Thus, we obtain that for any $T>0$ there exists a constant $C=C(T, \phi)$, depending on some constants of the problem (namely $\lambda_{1}, C_{g}$ and $f$ ), and on $T$ and $\phi$, such that

$$
\left|u_{t}^{m}\right|_{C_{H}}^{2} \leq C(T, \phi) \quad \forall t \in[0, T], \quad \forall m \geq 1 .
$$

In particular, this implies that $t_{m}=\infty$ for all $m$, and taking into account that $u^{m}(s)=P_{m} \phi(s)$ for $s \in[-h, 0]$,

$$
\text { the sequence }\left\{u^{m}\right\} \text { is bounded in } L^{\infty}(-h, T ; H) \quad \forall T>0 \text {. }
$$

Moreover, it follows from (9), g3) and (13) that

$$
\text { the sequence }\left\{u^{m}\right\} \text { is bounded in } L^{2}(0, T ; V) \quad \forall T>0 \text {. }
$$

On the other hand, multiplying (8) by $\frac{d}{d t} \alpha_{m_{j}}(t)$, summing in $j$, and integrating over $\Omega$, we have

$$
\frac{1}{2} \frac{d}{d t}\left\|u^{m}(t)\right\|^{2}+\left|\frac{d}{d t} u^{m}\right|^{2}+\left\|\frac{d}{d t} u^{m}\right\|^{2}=\left(g\left(t, u_{t}^{m}\right), \frac{d}{d t} u^{m}\right) .
$$

Now, on account of the Young inequality,

$$
\frac{d}{d t}\left\|u^{m}(t)\right\|^{2}+\left|\frac{d}{d t} u^{m}\right|^{2}+2\left\|\frac{d}{d t} u^{m}\right\|^{2} \leq C_{g}\left|u_{t}^{m}\right|_{C_{H}}^{2}+f(t) .
$$

Integrating (16) from 0 to $t$, we deduce

$$
\begin{aligned}
\left\|u^{m}(t)\right\|^{2} & +\int_{0}^{t}\left|\frac{d}{d s} u^{m}\right|^{2} d s+2 \int_{0}^{t}\left\|\frac{d}{d s} u^{m}\right\|^{2} d s \\
& \leq\left\|u^{m}(0)\right\|^{2}+\int_{0}^{t}\left(C_{g}\left|u_{s}^{m}\right|_{C_{H}}^{2}+f(s)\right) d s .
\end{aligned}
$$

Thanks to (15), (17) and the fact that $\left\|u^{m}(0)\right\|=\left\|P_{m} \phi(0)\right\| \leq\|\phi(0)\|$, the sequence of time derivatives

$$
\left\{\frac{d}{d t}\left(u^{m}\right)\right\} \text { is bounded in } L^{2}(0, T ; V), \quad \forall T>0 .
$$

Thus, this implies the existence of a function $u \in L^{\infty}(-h, T ; H) \cap L^{2}(0, T ; V)$, with $u^{\prime} \in L^{2}\left(0, T ; V^{\prime}\right)$, for all $T>0$, and a subsequence of $\left\{u^{m}\right\}$ which converges weak-star to $u$ in $L^{\infty}(-h, T ; H)$ and weakly to $u$ in $L^{2}(0, T ; V)$, with $\left\{\left(u^{m}\right)^{\prime}\right\}$ converging weakly to $u^{\prime}$ in $L^{2}\left(0, T ; V^{\prime}\right)$ for all $T>0$. Observe that, in particular, $u \in C([0, \infty) ; H)$.

By the Aubin-Lions compactness result (cf. [5, Ch.1,Th.5.1]), we obtain that a subsequence in fact converges strongly to $u$ in $L^{2}(0, T ; H)$ and a.e. in $(0, T)$ with values in $H$, and a.e. in $(0, T) \times \Omega$ for all $T>0$.

\section{Step 2: Convergence in $C_{H}$ and existence of solution.}

We will prove that $u_{t}^{m} \rightarrow u_{t}$ in $C_{H}$, for all $t \in[0, \infty)$. To see this, it is enough to prove:

$$
\begin{aligned}
& P_{m} \phi \rightarrow \phi \quad \text { in } C_{H}, \\
& u^{m} \rightarrow u \text { in } C([0, T] ; H), \forall T>0 .
\end{aligned}
$$


For the delay initial datum $\phi \in C_{H}$, if (19) were not true, there would exist $\varepsilon>0$ and a subsequence, that we would relabel the same, such that

$$
\left|P_{m} \phi\left(\theta_{m}\right)-\phi\left(\theta_{m}\right)\right|>\varepsilon \quad \forall m \geq 1 \text {. }
$$

One can assume that $\theta_{m} \rightarrow \theta$, where $\theta \in[-h, 0]$. Then $P_{m} \phi\left(\theta_{m}\right) \rightarrow \phi(\theta)$, since $\left|P_{m} \phi\left(\theta_{m}\right)-\phi(\theta)\right| \leq\left|P_{m} \phi\left(\theta_{m}\right)-P_{m} \phi(\theta)\right|+\left|P_{m} \phi(\theta)-\phi(\theta)\right| \rightarrow 0$ as $m \rightarrow \infty$. But this is a contradiction with (21) and the continuity of $\phi$. Therefore, (19) holds.

Now we consider an arbitrary fixed value $T>0$. Due to the strong convergence of $\left\{u^{m}\right\}$ to $u$ in $L^{2}(0, T ; H)$, we deduce that a subsequence (relabelled again as $u^{m}$ ) converges to $u(t)$ in $H$ a.e. $t \in(0, T)$.

Since

$$
u^{m}(t)-u^{m}(s)=\int_{s}^{t}\left(u^{m}\right)^{\prime}(r) d r \quad \text { in } V^{\prime}, \forall s, t \in[0, T],
$$

from (18) (notice that a bounded sequence in

$$
L^{2}(0, T ; V)
$$

is also bounded in $\left.L^{2}\left(0, T ; V^{\prime}\right)\right)$ we have that $\left\{u^{m}\right\}$ is equi-continuous on $[0, T]$ with values in $V^{\prime}$. By the compactness of the injection of $H$ into $V^{\prime}$, from (14) and the equi-continuity in $V^{\prime}$, by the Ascoli-Arzelà theorem we have that

$$
u^{m} \rightarrow u \quad \text { in } C\left([0, T] ; V^{\prime}\right) .
$$

Again from (14) we obtain that for any sequence $\left\{t_{m}\right\} \subset[0, T]$, with $t_{m} \rightarrow t$, one has

$$
u^{m}\left(t_{m}\right) \rightarrow u(t) \quad \text { weakly in } H,
$$

where we have used (22) in order to identify what is the weak limit.

Now, we will prove (20) by a contradiction argument. If (20) were not true, then, taking into account that $u \in C([0, T] ; H)$, there would exist $\varepsilon>0$, a value $t_{0} \in[0, T]$ and subsequences (relabelled the same) $\left\{u^{m}\right\}$ and $\left\{t_{m}\right\} \subset[0, T]$ with $\lim _{m \rightarrow \infty} t_{m}=t_{0}$ such that

$$
\left|u^{m}\left(t_{m}\right)-u\left(t_{0}\right)\right| \geq \varepsilon \quad \forall m .
$$

In order to prove that this is a contradiction, we will use an energy method.

By (10) and (13), we have the following energy inequality for all $u^{m}$ (see also Remark 3.3):

$$
\begin{aligned}
\left|u^{m}(t)\right|^{2}+\left\|u^{m}(t)\right\|^{2} \leq & \left|u^{m}(s)\right|^{2}+\left\|u^{m}(s)\right\|^{2}+\left(2 \lambda_{1}\right)^{-1} C_{g} C(T, \phi)(t-s) \\
& +\left(2 \lambda_{1}\right)^{-1} \int_{s}^{t} f(r) d r, \quad \forall s, t \in[0, T] .
\end{aligned}
$$

On the other hand, we deduce from (13), and (g3), eventually extracting a subsequence, the existence of $\xi_{g} \in L^{2}(0, T ; H)$, such that $\left\{g\left(u^{m}\right)\right\}$ converges weakly to $\xi_{g}$ in $L^{2}(0, T ; H)$.

Then, taking into account (14), (15) and (18), we can pass to the limit in equation (8) and deduce that $u$ is solution of

$$
\begin{aligned}
\frac{d}{d t}(u(t), v)+\frac{d}{d t}((u(t), & v))+((u(t), v)) \\
& =\left(\xi_{g}(t), v\right), \quad \text { a.e. } t \in(0, T), \forall v \in V
\end{aligned}
$$


Therefore, by the energy equality and Young's inequality,

$$
\begin{aligned}
&|u(t)|^{2}+\|u(t)\|^{2}+2 \int_{s}^{t}\|u(r)\|^{2} d r \\
&=|u(s)|^{2}+\|u(s)\|^{2}+2 \int_{s}^{t}\left(\xi_{g}(r), u(r)\right) d r \\
& \leq|u(s)|^{2}+\|u(s)\|^{2}+2 \int_{s}^{t}\|u(r)\|^{2} d r \\
&+\left(2 \lambda_{1}\right)^{-1} \int_{s}^{t}\left|\xi_{g}(r)\right|^{2} d r, \quad \forall s, t \in[0, T] .
\end{aligned}
$$

Now, observe that, for the weak limit $\xi_{g}$, we have the estimate

$$
\begin{aligned}
\int_{s}^{t}\left|\xi_{g}(r)\right|^{2} d r & \leq \liminf _{m \rightarrow \infty} \int_{s}^{t}\left|g\left(r, u_{r}^{m}\right)\right|^{2} d r \\
& \leq C_{g} C(T, \phi)(t-s)+\int_{s}^{t} f(r) d r, \quad \forall 0 \leq s \leq t \leq T .
\end{aligned}
$$

Consequently, $u$ satisfies the inequality (25).

Now, consider the continuous functions $J_{m}, J:[0, T] \rightarrow \mathbb{R}$ defined by

$$
\begin{gathered}
J_{m}(t)=\frac{1}{2}\left(\left|u^{m}(t)\right|^{2}+\left\|u^{m}(t)\right\|^{2}\right)-\frac{1}{2 \lambda_{1}} \int_{0}^{t} f(r) d r-\frac{C_{g} C(T, \phi)}{2 \lambda_{1}} t, \\
J(t)=\frac{1}{2}\left(|u(t)|^{2}+\|u(t)\|^{2}\right)-\frac{1}{2 \lambda_{1}} \int_{0}^{t} f(r) d r-\frac{C_{g} C(T, \phi)}{2 \lambda_{1}} t .
\end{gathered}
$$

From (25) for $\left\{u^{m}\right\}$ and $u$, it is clear that $J_{m}$ and $J$ are non-increasing functions. Moreover, by the convergence of $u^{m}$ to $u$ a.e. $t \in(0, T)$ with values in $H$, and weakly in $L^{2}(0, T ; H)$, it holds that

$$
J_{m}(t) \rightarrow J(t) \quad \text { a.e. } t \in[0, T] .
$$

Now we will prove that

$$
u^{m}\left(t_{m}\right) \rightarrow u\left(t_{0}\right) \quad \text { in } H
$$

which contradicts (24).

Firstly, recall from (23) that

$$
u^{m}\left(t_{m}\right) \rightarrow u\left(t_{0}\right) \quad \text { weakly in } H .
$$

So, we have that

$$
\left|u\left(t_{0}\right)\right| \leq \liminf _{m \rightarrow \infty}\left|u^{m}\left(t_{m}\right)\right| .
$$

Therefore, if we show that

$$
\limsup _{m \rightarrow \infty}\left|u^{m}\left(t_{m}\right)\right| \leq\left|u\left(t_{0}\right)\right|,
$$

we obtain that $\lim _{m \rightarrow \infty}\left|u^{m}\left(t_{m}\right)\right|=\left|u\left(t_{0}\right)\right|$, which jointly with (29), implies (28).

Now, observe that the case $t_{0}=0$ follows directly from (25) with $s=0$ and the definition of $u^{m}(0)=P_{m} \phi(0)$. So, we may assume that $t_{0}>0$. This is important, since we will approach this value $t_{0}$ from the left by a sequence $\left\{\tilde{t}_{k}\right\}$, i.e. $\lim _{k \rightarrow \infty} \tilde{t}_{k} \nearrow t_{0}$, being $\left\{\tilde{t}_{k}\right\}$ values where $(27)$ holds. Since $u(\cdot)$ is continuous at $t_{0}$, there is $k_{\varepsilon}$ such that

$$
\left|J\left(\tilde{t}_{k}\right)-J\left(t_{0}\right)\right|<\varepsilon / 2, \quad \forall k \geq k_{\varepsilon} .
$$


On the other hand, taking $m \geq m\left(k_{\varepsilon}\right)$ such that $t_{m}>\tilde{t}_{k_{\varepsilon}}$, as $J_{m}$ is non-increasing and for all $\tilde{t}_{k}$ the convergence (27) holds, one has that

$$
J_{m}\left(t_{m}\right)-J\left(t_{0}\right) \leq\left|J_{m}\left(\tilde{t}_{k_{\varepsilon}}\right)-J\left(\tilde{t}_{k_{\varepsilon}}\right)\right|+\left|J\left(\tilde{t}_{k_{\varepsilon}}\right)-J\left(t_{0}\right)\right|,
$$

and obviously, taking $m \geq m^{\prime}\left(k_{\varepsilon}\right)$, it is possible to obtain $\left|J_{m}\left(\tilde{t}_{k_{\varepsilon}}\right)-J\left(\tilde{t}_{k_{\varepsilon}}\right)\right|<\varepsilon / 2$. We also have that

$$
\int_{0}^{t_{m}} f(r) d r \rightarrow \int_{0}^{t_{0}} f(r) d r
$$

which implies that (30) holds. Thus, (28) and finally (20) also hold true.

Now, we can pass to the limit in the equations satisfied by $\left\{u^{m}\right\}$ and complete the information obtained in (26).

The first straightforward consequence of the convergence proved above, since $g$ satisfies (g2), is that

$$
g\left(\cdot, u^{m}\right) \rightarrow g(\cdot, u .) \quad \text { in } L^{2}(0, T ; H), \quad \forall T>0 .
$$

Thus, we can identify $\xi_{g}(t)=g\left(t, u_{t}\right)$ in (26). Therefore $u$ is a solution of (1).

\section{Uniqueness}

Let $u, v$ be two weak solutions with the same initial conditions and set $w=v-u$. Then, using the energy equality, we obtain

$$
\frac{1}{2} \frac{d}{d t}\left(|w|^{2}+\|w\|^{2}\right)+\|w\|^{2}=\left(g\left(t, u_{t}\right)-g\left(t, v_{t}\right), w\right), \quad t \in(0, T) .
$$

On the other hand, reasoning as in (12), we deduce that

$$
\left|u_{t}\right|_{C_{H}}^{2} \leq\left(|\phi|_{C_{H}}^{2}+\|\phi(0)\|+\left(2 \lambda_{1}\right)^{-1} \int_{0}^{T} f(s) d s\right) e^{C_{g}\left(2 \lambda_{1}\right)^{-1} T}
$$

for all $t \in[0, T]$, and the same for $v$.

This inequality and g2), yield that

$$
\begin{aligned}
\frac{d}{d t}\left(|w(t)|^{2}+\|w(t)\|^{2}\right) & \leq 2 L_{g}\left(R_{T, \phi}\right)\left|w_{t}\right|_{C_{H}}|w(t)| \\
& \leq 2 L_{g}\left(R_{T, \phi}\right)\left|w_{t}\right|_{C_{H}}^{2},
\end{aligned}
$$

for all $t \in[0, T]$.

Observe that $w(s)=0$ if $s \leq \tau$. Therefore, for $t \in(0, T)$ :

$$
\left|w_{t}\right|_{C_{H}}^{2} \leq 2 L_{g}\left(R_{T, \phi}\right) \int_{\tau}^{t}\left|w_{s}\right|_{C_{H}}^{2} d s,
$$

whence the Gronwall lemma finishes the proof.

Proposition 3.5. Under the assumptions of Theorem 3.4, the solution of (1) is continuous with respect to the initial condition $\phi$. More precisely, if $u^{i}$, for $i=1,2$, are the corresponding solutions to the initial data $\phi^{i} \in C_{H}, i=1,2$, with $\phi^{i}(0) \in V$, $i=1,2$, the following estimate holds:

$$
\left|u_{t}^{1}-u_{t}^{2}\right|_{C_{H}}^{2} \leq\left(\left|\phi^{1}-\phi^{2}\right|_{C_{H}}^{2}+\left\|\phi^{1}(0)-\phi^{2}(0)\right\|^{2}\right) e^{2 L_{g}\left(R_{T, \phi_{1}, \phi_{2}}\right) t},
$$

for all $t \in[\tau, T]$, where $R_{T, \phi_{1}, \phi_{2}} \geq 0$ is given by

$$
R_{T, \phi_{1}, \phi_{2}}^{2}=\left(\max \left(\left|\phi^{1}\right|_{C_{H}}^{2},\left|\phi^{2}\right|_{C_{H}}^{2}\right)+\left(2 \lambda_{1}\right)^{-1} \int_{0}^{T} f(s) d s\right) e^{C_{g}\left(2 \lambda_{1}\right)^{-1} T} .
$$


Proof. We denote $w=u^{1}-u^{2}$. It easily follows that

$$
\frac{d}{d t}\left(|w|^{2}+\|w\|^{2}\right)+2\|w\|^{2}=2\left(g\left(t, u_{t}\right)-g\left(t, v_{t}\right), w\right), \quad t \in(\tau, T)
$$

For a fixed $T>0$, we know that $u$ and $v$ belong to $C(-h, T ; H)$, thus there exists $R_{T, \phi_{1}, \phi_{1}}>0$ such that $\left|u_{t}\right|_{C_{H}} \leq R_{T, \phi_{1}, \phi_{1}}$ and $\left|v_{t}\right|_{C_{H}} \leq R_{T, \phi_{1}, \phi_{1}}$, for all $t \in[\tau, T]$.

Then, by (g2) and the Young inequality,

$$
\begin{aligned}
|w(t)|^{2}+\|w(t)\|^{2} \leq & |w(\tau)|^{2}+\|w(\tau)\|^{2}+2 L_{g}\left(R_{T, \phi_{1}, \phi_{2}}\right) \int_{\tau}^{t}\left|w_{s}\right|_{C_{H}}|w(s)| d s \\
\leq & \left|\phi_{1}-\phi_{2}\right|_{C_{H}}^{2}+\left\|\phi_{1}(0)-\phi_{2}(0)\right\|^{2} \\
& +2 L_{g}\left(R_{T, \phi_{1}, \phi_{2}}\right) \int_{\tau}^{t}\left|w_{s}\right|_{C_{H}}^{2} d s
\end{aligned}
$$

for all $t \in[\tau, T]$.

Thus,

$$
\left|w_{t}\right|_{C_{H}}^{2} \leq\left|\phi^{1}-\phi^{2}\right|_{C_{H}}^{2}+\left\|\phi_{1}(0)-\phi_{2}(0)\right\|^{2}+2 L_{g}\left(R_{T, \phi_{1}, \phi_{2}}\right) \int_{\tau}^{t}\left|w_{s}\right|_{C_{H}}^{2} d s
$$

for all $t \in[\tau, T]$, and therefore, thanks to the Gronwall lemma, we deduce (31).

\section{Stationary solutions and their stability}

In this section we will prove that, under additional assumptions, there exists a unique stationary solution of problem (1) which is globally asymptotically exponentially stable.

From now on we assume that $g: \mathbb{R}_{+} \times C_{H} \rightarrow H$ satisfies g1)-g3) with $f(t)=$ $|f| \geq 0$ for all $t \geq 0$, a constant function.

We also suppose that $g$ is autonomous, in the sense that there exists a function $g_{0}: H \rightarrow H$ such that

g4) $g(t, w)=g_{0}(w)$ for all $(t, w) \in[0, \infty) \times H$,

where, with a slight abuse of notation, we identify every element $w \in H$ with the constant function in $C_{H}$ which is equal to $w$ for any time $t \in[-h, 0]$.

We consider the following equation,

$$
\frac{d}{d t}(u+A u)+A u=g\left(t, u_{t}\right) \quad t>0 .
$$

A stationary solution to (32) will be an element $u^{*} \in V$ such that

$$
\left(\left(u^{*}, v\right)\right)=\left(g_{0}\left(u^{*}\right), v\right) \quad \forall v \in V, \forall t>0 .
$$

THEOREM 4.1. Under the above assumptions and notation, if $\lambda_{1}>C_{g}^{1 / 2}$, then:

(a) The problem (32) admits at least one stationary solution $u^{*}$ (which indeed belongs to $D(A))$. Moreover, any such stationary solution satisfies the estimate

$$
\left(\lambda_{1}-C_{g}^{1 / 2}\right)\left\|u^{*}\right\| \leq \lambda_{1}^{1 / 2}|f|^{1 / 2} .
$$

(b) If

$$
\lambda_{1}>L_{g}\left(R_{g}\right)
$$


where

$$
R_{g}=\frac{|f|^{1 / 2}}{\lambda_{1}-C_{g}^{1 / 2}},
$$

then, the stationary solution of (32) is unique.

Proof. First, we will obtain the estimate (34). If $u^{*}$ is a stationary solution, it must verify

$$
\left(\left(u^{*}, v\right)\right)=\left(g_{0}\left(u^{*}\right), v\right) \quad \forall v \in V, t>0
$$

and, therefore,

$$
\begin{aligned}
\left\|u^{*}\right\|^{2} & \leq\left|g_{0}\left(u^{*}\right)\right|\left|u^{*}\right| \\
& \leq\left(C_{g}^{1 / 2}\left|u^{*}\right|+|f|^{1 / 2}\right)\left|u^{*}\right| \\
& \leq \lambda_{1}^{-1} C_{g}^{1 / 2}\left\|u^{*}\right\|^{2}+\lambda_{1}^{-1 / 2}|f|^{1 / 2}\left\|u^{*}\right\| .
\end{aligned}
$$

As for the existence, let us consider $\left\{v_{j}\right\} \subset V$, the orthonormal basis of $H$ of all the eigenfunctions of the operator $A$. For each integer $m \geq 1$, let us denote again $V_{m}=\operatorname{span}\left[v_{1}, \ldots, v_{m}\right]$, with the inner product $((\cdot, \cdot))$ and norm $\|\cdot\|$. Define the operators $R_{m}: V_{m} \rightarrow V_{m}, m \geq 1$, by

$$
\left(\left(R_{m} u, v\right)\right)=((u, v))-\left(g_{0}(u), v\right), \quad \forall u, v \in V_{m} .
$$

Since the right hand side is a continuous linear map from $V_{m}$ to $\mathbb{R}$, by the Riesz theorem, each $R_{m} u \in V_{m}$ is well defined. We check now that $R_{m}$ is continuous.

$$
\begin{aligned}
\left(\left(R_{m} u-R_{m} \widetilde{u}, v\right)\right) & =((u-\widetilde{u}, v))-\left(g_{0}(u)-g_{0}(\widetilde{u}), v\right) \\
& \leq\|u-\widetilde{u}\|\|v\|+\lambda_{1}^{-1} L_{g}(R)\|u-\widetilde{u}\|\|v\| \\
& \leq\left(1+\lambda_{1}^{-1} L_{g}(R)\right)\|u-\widetilde{u}\|\|v\|,
\end{aligned}
$$

for all $u, \widetilde{u}, v \in V_{m}$, where $R=\max \{|u|,|\widetilde{u}|\}$.

Therefore,

$$
\left\|R_{m} u-R_{m} \widetilde{u}\right\| \leq\left(1+\lambda_{1}^{-1} L_{g}(R)\right)\|u-\widetilde{u}\|
$$

for all $u, \widetilde{u}$.

On the other hand, for all $u \in V_{m}$,

$$
\begin{aligned}
\left(\left(R_{m} u, u\right)\right) & =((u, u))-\left(g_{0}(u), u\right) \\
& \geq\|u\|^{2}-\lambda_{1}^{-1 / 2}|f|^{1 / 2}\|u\|-\lambda_{1}^{-1} C_{g}^{1 / 2}\|u\|^{2} .
\end{aligned}
$$

Thus, if we take

$$
\beta=\frac{\lambda_{1}^{-1 / 2}|f|^{1 / 2}}{1-C_{g} \lambda_{1}^{-1}},
$$

we obtain $\left(\left(R_{m} u, u\right)\right) \geq 0$ for all $u \in V_{m}$ such that $\|u\|=\beta$.

Consequently, by a corollary of the Brouwer fixed point theorem (see [5, p. $53]$ ), for each $m \geq 1$ there exist $u_{m} \in V_{m}$ such that $R_{m}\left(u_{m}\right)=0$, with $\left\|u_{m}\right\| \leq \beta$.

Observe moreover that $A u_{m} \in V_{m}$, and therefore

$$
\begin{aligned}
\left|A u_{m}\right|^{2} & =\left(g_{0}\left(u_{m}\right), A u_{m}\right) \\
& \leq \frac{1}{2}\left|A u_{m}\right|^{2}+\frac{|f|}{2}+\frac{C_{g} \beta^{2}}{2 \lambda_{1}} .
\end{aligned}
$$


From (39), we deduce that the sequence $\left\{u_{m}\right\}$ is bounded in $D(A)$, and consequently, by the compact injection of $D(A)$ in $V$, we can extract a subsequence $\left\{u_{m^{\prime}}\right\} \subset\left\{u_{m}\right\}$, which converges, weakly in $D(A)$ and strongly in $V$, to an element $u^{*} \in D(A)$. It is now standard to take limits in (37) and to obtain that $u^{*}$ is a stationary solution.

\section{Uniqueness}

Let us suppose that $u^{*}$ and $\widetilde{u}^{*}$ are two stationary solutions of (32). Then,

$$
\left(\left(u^{*}-\widetilde{u}^{*}, v\right)\right)=\left(g_{0}\left(u^{*}\right)-g_{0}\left(\widetilde{u}^{*}\right), v\right), \quad \forall v \in V, t>0 .
$$

Taking $v=u^{*}-\widetilde{u}^{*}$ and proceeding as in (38) we obtain from (40)

$$
\left\|u^{*}-\widetilde{u}^{*}\right\|^{2} \leq \lambda_{1}^{-1} L_{g}\left(R_{g}\right)\left\|u^{*}-\widetilde{u}^{*}\right\|^{2},
$$

where $R_{g}$ is the constant defined in (36).

Then, it is obvious that $u^{*}=\widetilde{u}^{*}$ if the condition $\lambda_{1}>L_{g}\left(R_{g}\right)$ is satisfied.

THEOREM 4.2. Assume that g1)-g4) hold with $f$ being time-independent and $\lambda_{1}>C_{g}^{1 / 2}$. Assume also that (35) is fulfilled. Let $u^{*}$ be the unique stationary solution of (32). The following facts hold:

a) If $L_{g}(R)=L_{g}$ is independent of $R$ (and therefore $\lambda_{1}>L_{g}$ ), there exist two constants $\lambda>0$ and $C_{\lambda}>0$ such that for any $\phi \in C_{H}$

$$
\left|u(t ; \phi)-u^{*}\right|^{2} \leq C_{\lambda}\left|\phi-u^{*}\right|_{C_{H}}^{2} e^{-\lambda t} \quad \text { for all } t \geq 2 h .
$$

b) Assume that $L_{g}(R)$ is a continuous function of $R$, and there exists $0<$ $\mu<\frac{2 \lambda_{1}}{\lambda_{1}+1}$ such that $\mu\left(2 \lambda_{1}-\mu \lambda_{1}-\mu\right) e^{-\mu h}>C_{g}$, and

$$
\lambda_{1}>L_{g}\left(\widetilde{R}_{g}\right)
$$

where $\widetilde{R}_{g}$ is the positive number given by

$$
\widetilde{R}_{g}^{2}=\max \left\{e^{\mu h}\left(2 \lambda_{1}-\mu \lambda_{1}-\mu\right)^{-1}\left(\mu-\left(2 \lambda_{1}-\mu \lambda_{1}-\mu\right)^{-1} e^{\mu h} C_{g}\right)^{-1}|f|, R_{g}^{2}\right\},
$$

with $R_{g}$ defined by (36). Then, there exists a constant $\lambda>0$ such that, for each $\phi \in C_{H}$ with $\phi(0) \in V$, there exists $T_{\phi} \geq 2 h$ and $C_{\lambda, \phi}>0$ such that

$$
\left|u(t ; \phi)-u^{*}\right|^{2} \leq C_{\lambda, \phi}\left|\phi-u^{*}\right|_{C_{H}}^{2} e^{-\lambda t} \quad \text { for all } t \geq T_{\phi}
$$

Proof. Let $\phi \in C_{H}$, and let $u(t)=u(t ; \phi)$ be the corresponding solution of (1). Let us also denote $w(t)=u(t)-u^{*}$. Considering equations (32) for $u(t)$ and (33) for $u^{*}$, we have

$$
\frac{d}{d t}[(w(t), v)+((w(t), v))]+((w(t), v))=\left(g\left(t, u_{t}\right)-g\left(t, u^{*}\right), v\right),
$$

a.e. $t>0$, for any $v \in V$.

For any $\lambda>0$,

$$
\begin{aligned}
\frac{d}{d t}\left(e^{\lambda t}\left[|w(t)|^{2}+\|w(t)\|^{2}\right]\right) \leq & e^{\lambda t}\left(\lambda\left[|w(t)|^{2}+\|w(t)\|^{2}\right]-\|w(t)\|^{2}\right. \\
& \left.+\left|g\left(t, u_{t}\right)-g\left(t, u^{*}\right) \| w(t)\right|\right),
\end{aligned}
$$

a.e. $t>0$.

Now, we consider two cases: 
Case a): Assume that $g$ is globally Lipschitz, i.e., $L_{g}(R)=L_{g}$ is independent of $R$.

In this case, from (44) and the Young inequality, we conclude that

$$
\frac{d}{d t}\left(e^{\lambda t}\left[|w(t)|^{2}+\|w(t)\|^{2}\right]\right) \leq e^{\lambda t}\left(\lambda-1+\lambda \lambda_{1}^{-1}\right)\|w(t)\|^{2}+L_{g} e^{\lambda t}\left|w_{t}\right|_{C_{H}}^{2},
$$

a.e. $t>0$, for any $\lambda>0$.

Therefore, integrating from $2 h$ to $t$, and observing that

$$
\sup _{r \in[s-h, s]}|w(r)|^{2} \leq \lambda_{1}^{-1} \sup _{r \in[h, s]}\|w(r)\|^{2}, \quad \text { for all } s \geq 2 h,
$$

we have

$$
\begin{aligned}
e^{\lambda t}|w(t)|^{2} \leq & e^{2 \lambda h}\left[|w(2 h)|^{2}+\|w(2 h)\|^{2}\right] \\
& +\left(\lambda-1+\lambda \lambda_{1}^{-1}+\lambda_{1}^{-1} L_{g}\right) \int_{2 h}^{t} e^{\lambda r} \sup _{r \in[h, s]}\|w(r)\|^{2} d s
\end{aligned}
$$

for all $t \geq 2 h$ and any $\lambda>0$.

Thus, taking into account the uniqueness condition of the Theorem 4.1, and taking $\lambda=\frac{\lambda_{1}-L_{g}}{1+\lambda_{1}}>0$, we obtain

$$
e^{\lambda t}|w(t)|^{2} \leq e^{2 \lambda h}\left[|w(2 h)|^{2}+\|w(2 h)\|^{2}\right] \quad \text { for all } t \geq 2 h .
$$

Hence, by Proposition 3.5, we deduce (41) with $C_{\lambda}=e^{2\left(\lambda+2 L_{g}\right) h}$.

Case b): Now, the Young inequality once again implies

$$
\begin{aligned}
\frac{d}{d t}\left(e^{\mu t}\left[|u(t)|^{2}+\|u(t)\|^{2}\right]\right) \leq & \mu e^{\mu t}\left(|u(t)|^{2}+\|u(t)\|^{2}\right) \\
& +e^{\mu t}\left(-2\|u(t)\|^{2}+2\left|g\left(t, u_{t}\right) \| u(t)\right|\right) \\
\leq & e^{\mu t}\left(\mu \lambda_{1}^{-1}+\mu-2\right)\|u(t)\|^{2}+2 e^{\mu t}\left|g\left(t, u_{t}\right) \| u(t)\right| \\
\leq & e^{\mu t} \lambda_{1}^{-1}\left(2-\mu \lambda_{1}^{-1}-\mu\right)^{-1}\left|g\left(t, u_{t}\right)\right|^{2},
\end{aligned}
$$

a.e. $t>0$, and therefore, by g3),

$$
\begin{aligned}
\frac{d}{d t}\left(e^{\mu t}\left[|u(t)|^{2}+\|u(t)\|^{2}\right]\right) \leq & C_{g}\left(2 \lambda_{1}-\mu \lambda_{1}-\mu\right)^{-1} e^{\mu t}\left|u_{t}\right|_{C_{H}}^{2} \\
& +\left(2 \lambda_{1}-\mu \lambda_{1}-\mu\right)^{-1}|f| e^{\mu t} \text { a.e. } t>0 .
\end{aligned}
$$

Integrating this last inequality, we obtain

$$
\begin{aligned}
e^{\mu t}\left[|u(t)|^{2}+\|u(t)\|^{2}\right] \leq & {\left[|\phi(0)|^{2}+\|\phi(0)\|^{2}\right]+\left(2 \lambda_{1}-\mu \lambda_{1}-\mu\right)^{-1}|f| \int_{0}^{t} e^{\mu s} d s } \\
& +C_{g}\left(2 \lambda_{1}-\mu \lambda_{1}-\mu\right)^{-1} \int_{0}^{t}\left|u_{s}\right|_{C_{H}}^{2} e^{\mu s} d s
\end{aligned}
$$

for all $t \geq 0$.

Now,

$$
\begin{aligned}
e^{\mu t}\left|u_{t}\right|_{C_{H}}^{2} & =e^{\mu t} \sup _{s \in[-h, 0]}|u(t+s)|^{2} \\
& =e^{\mu t} \sup _{\theta \in[t-h, t]}|u(\theta)|^{2} \\
& =\sup _{\theta \in[t-h, t]} e^{\mu \theta}|u(\theta)|^{2} e^{\mu(t-\theta)} \\
& \left.\leq e^{\mu h} \sup _{\theta \in[t-h, t]}\right]^{\mu \theta}|u(\theta)|^{2}
\end{aligned}
$$


Thus, it is easy to deduce that

$$
\begin{aligned}
e^{\mu t}\left|u_{t}\right|_{C_{H}}^{2} \leq & e^{\mu h}\left(|\phi|_{C_{H}}^{2}+\|\phi(0)\|^{2}\right)+\left(2 \lambda_{1}-\mu \lambda_{1}-\mu\right)^{-1}|f| e^{\mu h} \int_{0}^{t} e^{\mu s} d s \\
& +C_{g}\left(2 \lambda_{1}-\mu \lambda_{1}-\mu\right)^{-1} e^{\mu h} \int_{0}^{t}\left|u_{s}\right|_{C_{H}}^{2} e^{\mu s} d s
\end{aligned}
$$

for all $t \geq 0$, and therefore, thanks to the Gronwall lemma, we deduce

$$
\begin{aligned}
& \left|u_{t}\right|_{C_{H}}^{2} \\
& \quad \leq\left\{\left(|\phi|_{C_{H}}^{2}+\|\phi(0)\|^{2}+\left(2 \lambda_{1}-\mu \lambda_{1}-\mu\right)^{-1} \mu^{-1}|f|\right) e^{\left(\left(2 \lambda_{1}-\mu \lambda_{1}-\mu\right)^{-1} e^{\mu h} C_{g}-\mu\right) t}\right. \\
& \left.\quad+\left(2 \lambda_{1}-\mu \lambda_{1}-\mu\right)^{-1}\left(\mu-\left(2 \lambda_{1}-\mu \lambda_{1}-\mu\right)^{-1} e^{\mu h} C_{g}\right)^{-1}|f|\right\} e^{\mu h},
\end{aligned}
$$

for all $t \geq 0$.

By (42) and the continuity of $L_{g}$, there exists an $\varepsilon>0$ such that

$$
\lambda_{1}>L_{g}\left(\widetilde{R}_{g}+\varepsilon\right)
$$

and a $T_{\phi} \geq 2 h$ such that

$$
\left|u_{t}\right|_{C_{H}} \leq \widetilde{R}_{g}+\varepsilon \quad \forall t \geq T_{\phi}
$$
obtain

Reasoning as in case a), we can prove that if we take $\lambda=\frac{\lambda_{1}-L_{g}\left(\widetilde{R}_{g}+\varepsilon\right)}{1+\lambda_{1}}>0$, we

$$
e^{\lambda t}|w(t)|^{2} \leq e^{\lambda T_{\phi}}\left[\left|w\left(T_{\phi}\right)\right|^{2}+\left\|w\left(T_{\phi}\right)\right\|^{2}\right] \quad \text { for all } t \geq T_{\phi} .
$$

Thus, arguing as in the proof of Proposition 3.5, we deduce (43) with $C_{\lambda, \phi}=$ $e^{\left(\lambda+2 L_{g}\left(\widetilde{R}_{g}+\varepsilon\right)\right) T_{\phi}}$.

Conclusions. We have proved some results on the existence, uniqueness and asymptotic behaviour of the solutions for a nonclassical diffusion equation with delay forcing term. Our assumptions are general enough to include several types of delay in the formulation (constant delay, variable delay with only measurable delay function, distributed delay, etc.). In particular, we have analyzed the exponential stability of the stationary solutions. The existence of pullback attractors in the case of differentiable delay with additional assumptions has been recently considered in [4], so it will be also interesting to carry out a similar global analysis of this model by proving the existence of attractors as well as the study of their geometrical structure by imposing only measurability on the delay function as well as considering more general types of delay terms. We plan to investigate these issues in a subsequent paper.

\section{References}

[1] C.T. Anh and T.Q. Bao, Pullback attractors for a class of non-autonomous nonclassical diffusion equations, Nonlinear Analysis 73 (2010), 399412.

[2] E.C. Aifantis, On the problem of diffusion in solids, Acta Mech. 37 (1980), 265296.

[3] J.K. Hale and S.M. Verduyn Lunel, "Introduction to Functional Differential Equations", Springer Verlag, New York, 1993.

[4] Z. Hu and Y. Wang, Pullback attractors for a nonautonomous nonclassical diffusion equation with variable delay, J. Math. Phys. 53, 072702 (2012) 
[5] J. L. Lions, "Quelques méthodes de résolution des problèmes aux limites non linéaires", Dunod, Gauthier-Villars, Paris, 1969.

[6] J.C. Peter, M.E. Gurtin, On a theory of heat conduction involving two temperatures, $Z$. Angew. Math. Phys. 19 (1968) 614627

[7] F. Rivero, Time dependent perturbation in a non-autonomous non-classical parabolic equation, Discrete Contin. Dyn. Syst. Ser. B 18 (2013), no. 1, 209221.

[8] T.W. Ting, Certain non-steady flows of second-order fluids, Arch. Ration. Mech. Anal. 14 (1963), 126.

[9] C. Truesdell and W. Noll, "The Nonlinear Field Theories of Mechanics", in: Encyclopedia of Physics, Springer, Berlin, 1995.

[10] C. Sun and M. Yang, Dynamics of the nonclassical diffusion equations, Asymptotic Analysis 59 (2008), 5181.

[11] C. Sun, S. Wang and C. Zhong, Global attractors for a nonclassical diffusion equation, Acta Math. Appl. Sin. Engl. Ser 23 (2007), 12711280.

Departamento de Ecuaciones Diferenciales y Análisis Numérico, Universidad de Sevilla, Apdo. De Correos 1160, 41080-Sevilla, SPAIN

E-mail address: caraball@us.es

Departamento de Ecuaciones Diferenciales y AnÁlisis Numérico, Universidad de Sevilla, Apdo. de Correos 1160, 41080-Sevilla, SPAin. Departamento de Economía, Métodos Cuantitativos e Historia Económica, Universidad Pablo de Olavide, Ctra. de Utrera, KM. 1, 41013-SEVIlla, SPAIN

E-mail address: ammardur@upo.es 